\title{
REVIEW
}

\section{Antimicrobial peptides and their potential application in inflammation and sepsis}

\author{
Tobias Schuerholz*, Klaus Brandenburg² and Gernot Marx \\ This article is one of eleven reviews selected from the Annual Update in Intensive Care and Emergency Medicine 2012 (Springer Verlag, \\ DOI: 10.1007/978-3-642-25716-2) and co-published as a series in Critical Care. Other articles in the series can be found online at \\ http://ccforum.com/series/annualupdate2012. Further information about the Annual Update in Intensive Care and Emergency Medicine is \\ available from http://www.springer.com/series/8901.
}

\section{Introduction}

Starting treatment early is key to increasing survival in patients with severe sepsis and septic shock. The crucial significance of timing has been demonstrated for the treatment of circulatory failure [1], use of antibiotics [2] and use of activated protein $C$ as adjunctive therapy [3]. Whereas it is of vital importance not only to begin antiinfective therapy as soon as possible but to also choose the adequate anti-infective drug [4], the impending problem is the growing number of multi-resistant bacteria [5]. Therefore, there is an increasing interest in the identification and development of new anti-infective agents.

Antimicrobial peptides (AMPs) are found in species ranging from bacteria and insects to mammals. They were identified over 100 years ago as an important part of innate immunity and can be isolated in body fluids and on body surfaces either constitutively or after inflammatory stimulation [6]. Compared to conventional antiinfective agents, some AMP may kill bacteria but also simultaneously neutralize released pathogenic factors, like lipopolysaccharide (LPS) or lipoprotein (LP), thus preventing the devastating consequences of the proinflammatory cascades in severe sepsis and septic shock. The obstacle in the application of naturally occurring AMPs is their high toxicity, promoting hemolysis, nephrotoxicity and neurotoxicity [7]. The challenge is, therefore, to develop synthetic peptide-based drugs on the basis of naturally occurring AMPs in order to effectively treat septic patients without causing harm.

*Correspondence: tschuerholz@ukaachen.de

'Department of Intensive Care, University Hospital, Pauwelsstr. 30, 52074 Aachen, Germany

Full list of author information is available at the end of the article

\section{Naturally occurring antimicrobial peptides in inflammation}

It has been realized for decades that AMPs have antiGram-positive and -negative effects [8] as well as antiviral and anti-yeast effects. These effects are limited by several mechanisms including modulation of the surface charge and the use of active extrusion [9-11]. Compared to resistance against AMPs, the bacterial mechanisms to defeat conventional anti-infective agents are more evident [12].

The majority of studies have addressed the so-called 'defensins', consisting of an alpha and beta subgroup. There are six different human alpha-defensins [6]. Three, highly homologous human defensins are most important: The human neutrophil peptides (HNP)1-3. HNP1-3 are stored in the azurophilic granules of polymorphonuclear leukocytes (PMN). HNP1-3 deliver approximately $5 \%$ of total PMN protein and comprise about $99 \%$ of the total defensin content of the neutrophils. Their antimicrobial activity is directed against bacteria (Gram-positive and Gram-negative) and viruses (herpes simplex virus [HSV], cytomegalovirus [CMV], human immunodeficiency virus [HIV]-1). HNP1-3 are chemotactic and regulate the release of cytokines and complement [6].

A comparable range of efficacy with potent antimicrobial activity against bacteria and fungi is described for LL-37. LL-37 is generated by the cleavage of hCAP18, the human cationic antimicrobial peptide of $18 \mathrm{kDa}$ and is isolated by neutrophils and epithelial tissues in respiratory, gastrointestinal and urogenital tracts [6].

The human beta-defensins (HBD)1-3 come from a variety of epithelial cells in different organs. HBD1-3 have broad antimicrobial activity. They are directed against bacteria, viruses and fungi, inducing chemokines and cytokines and thus recruiting cells of the adaptive immune system [6]. HBD3 downregulates pro-inflammatory 
cytokines, like tumor necrosis factor (TNF)- $\alpha$ or interleukin (IL)-6, in human and mouse macrophages after exposure to LPS in vitro and in vivo, suggesting a role for this defensin in the resolution of inflammatory processes. In contrast to other naturally occurring or synthetic peptides, this effect is not mediated through direct LPSpeptide LPS binding [13]. Deficits in the production of AMP usually expressed by human epithelial cells may lead to increased susceptibility to bacterial or viral infections [14]. These deficits may be caused by immunosuppressive drugs preventing the induction of AMP, such as HBD2, thus promoting infections [15]. The importance of HBD2 for protection against infections was demonstrated by Milner and Ortega who detected normal levels of HBD1 in burn patients, but the burn-associated loss of epithelium led to a decrease in HBD2 [16]. Since HBD2 effectively kills Escherichia coli, Staphylococcus aureus and other bacteria, reduced levels of this AMP may result in local bacterial proliferation [17].

Recently, these findings were confirmed in patients with multiple trauma [18]. The authors noted that despite open bone fractures and severe soft tissue trauma in multiple trauma, the rate of bacterial infection is surprisingly low and hypothesized that this may be related to serum concentrations of AMPs. Concentrations of HBD2, HBD3 and LL-37 were elevated after trauma suggesting a higher anti-bacterial effect compared to healthy donors thus explaining the relatively low infection rates [18].

Moreover, an increase or decrease in susceptibility to inflammation may be related to the highly variable interindividual composition of AMPs in body fluids [19]. Surprisingly, the capsular polysaccharide production in multiple Group A streptococci strains is upregulated by LL-37, thus increasing virulence [20]. LL-37 itself has functions other than chemotaxis, as shown by investigations of the effects of LL-37 on human omental arteries and veins [21]. In these studies, LL-37 induced endothelium-dependent relaxation by involvement of nitric oxide (NO) of endothelial origin. A localized increase in LL-37 can be mediated by degranulation of granulocytes following the process of granulocyte 'rolling' and 'sticking' on the endothelium [22]. Despite this possible negative consequence, therapeutic application of AMPs may have positive effects in a variety of inflammatory diseases. In experimental sepsis, the administration of AMP in certain doses was associated with increased survival [23]; the effective application of AMPs in animals was not confirmed in human trials. Iseganan, an analog of protegrin-1, a naturally occurring peptide with broadspectrum microbicidal activity, was not shown to be beneficial in reducing stomatitis in patients receiving chemotherapy [24].

In recent years, an increasing number of synthetic (cationic) peptides have been developed, but none has been approved for human use. Omiganan pentahydrochloride (synthetic cationic peptide; MBI 226), administered at insertion sites, was superior to povidone iodine in preventing central venous catheter-related bloodstream infections in a phase III trial; however, although the study was completed many years ago, the results have not been published in a peer-reviewed journal.

Local treatment of diabetic ulcer with AMPs had at least an equipotent anti-inflammatory effect compared to antibiotics [12]. In recent years, there have been at least 20 different peptides in several steps of development scheduled for anti-infective trials [23]. Two studies on human lactoferrin 1-11 peptide (hLF1-11) were aborted at Phase I and II stages. The purpose of these studies was to establish tolerance to treatment with hLF1-11 administered intravenously as a single daily dose for 10 consecutive days. The target population was patients with bacteremia due to Staphylococcus epidermidis (clinicaltrials.gov-identifier NCT00509847) or patients with proven candidemia (clinicaltrials.gov-identifier NCT00509834). The reasons given for withdrawal were that recruitment was not feasible within the timeframe (bacteremia) or the patient population was not available (candidemia).

\section{Development and anti-LPS effects of synthetic antimicrobial peptides}

Compounds to neutralize bacterial endotoxins were originally synthesized based on the binding region of the Limulus anti-LPS factor (LALF) [25]. Ried et al. were the first authors to report synthesis of peptides based on this domain. These authors found that the complete binding sequence, a cyclic peptide called cLALF22, had the greatest ability to bind to the lipid A part of LPS, its "endotoxic principle" [26]. Analogs shortened down to cLALF10 were much less active. In biophysical studies by Andrä et al., interaction of the complete LALF (called $\mathrm{ENP}=$ endotoxin-neutralizing protein in its recombinant form) and of part structures with endotoxins was studied, and considerable inhibition of the biological activity of LPS was found at protein [ENP]:[LPS] ratios greater than 20 to $200: 1$ molar [27-29].

Data on the supramolecular structure of LPS were indicative of a change of the lipid A cubic aggregate structure into a multilamellar one. This is of considerable interest, since it has been shown that the cubic aggregate structure of lipid A is its bioactive form, whereas some non-enterobacterial biologically inactive LPS/lipid A structures with a different acylation pattern adopt multilamellar aggregates [30]. In subsequent studies, LALFderived cyclic peptides were analyzed, starting with cLALF22 and various shortened analogs [27,29]. Consistently in all these investigations, a change in the endotoxin aggregate structure from a preferentially cubic into 
a multilamellar one was described, as well as a change in the morphologies of the LPS Re aggregates, as evidenced by freeze-fracture electron microscopy, from 'open eggshells' (i.e., spherical particles in the range 100 to $200 \mathrm{~nm}$ ), into large stacks of some $1000 \mathrm{~nm}$. The binding of the peptides to LPS was characterized in all cases as an exothermic process by isothermal titration calorimetry (ITC), driven by the Coulomb interaction of the positive charges of peptides with the negative charges of the endotoxins $[27,29]$.

Pan et al. synthesized the terminal part of the shrimp anti-LPS-factor, a peptide with 24 amino acids, in a cyclic and linear form. Pre-treatment of mice with the cyclic compound led to considerably enhanced survival of mice infected with approximately $10^{6}$ colony-forming units (cfu) of Pseudomonas aeruginosa. Parallel to this, the peptides were able to reduce the bacteria-induced production of TNF- $\alpha$ in the animals. However, already at a concentration of $2 \mu \mathrm{g} / \mathrm{ml}$ the peptides exhibited significant cytotoxicity in HeLa, MCF-7, and HT1080 cell lines, impeding their use as an anti-infective drug [31].

Because of the insufficient specificity of the above described peptides, we used a new approach, constructing a new series of peptides of amino acid lengths in the range 17 to 23 . The systematic study of various peptides differing in their amino acid chain lengths from 9 to 12 and 17 to 19 showed that the basic sequence, as given by Pep9 (FRRLKWKFW), was already able to confer significant inhibition of LPS-induced cytokine production. Relatively high concentrations of the peptides, however, were still necessary to inhibit cytokine production. Therefore, longer peptides were constructed to adapt to the physico-chemistry of the lipid A part of endotoxins. For this process, the composition of the $\mathrm{N}$-terminal side of the peptide was preferentially provided by polar and basic amino acids, and the $\mathrm{C}$-terminal by more hydrophobic ones. Furthermore, the exact number and type and sequence of amino acids was shown to be important, as deduced from the comparison of the peptides with different chain lengths [32-35].

Additional improvements were obtained by constructing the amino acid sequences on the basis of optimal binding to the lipid A moiety of bacterial LPS. Modifications of the sequences (for amino acid sequences see Table 1) led to a nearly complete inhibition of LPS-induced cytokine secretion at a [Pep]:[LPS] 3:1 molar ratio [33, 34]. This was particularly evident in the case of the lead structure, Pep19-2.5, which protected mice in a model of sepsis already at a [Pep]:[LPS] ratio of 50:1 weight\% (1250 ng peptide and 25 ng LPS).

More experiments were performed in an in vitro assay of human mononuclear cells, studying the peptideinduced inhibition of TNF- $\alpha$ induction by LPS, alone and in the presence of common antibiotics as a model for
Table 1. Synthetic antimicrobial peptide sequences and molecular weights

\begin{tabular}{lll}
\hline Pep19-2.5 & GCKKYRRFRWKFKGKFWFWG & 2711 \\
Pep19-2.5Dup & GWFWFKGKFKWRRRRYKKCG- & 5405 \\
& GCKKYRRFRWKFKGKFWFWG & \\
Pep19-2.5short & GCKKYRRFRWKFKGK & 1988 \\
\hline
\end{tabular}

combination therapy in septic patients. The data for the peptide alone (Figure 1) were indicative of the strong inhibition capacity of Pep19-2.5, whereas the antibiotic alone (streptomycin, see Figure 2) showed nearly no action. The combination of streptomycin with Pep19-2.5 at a 1:1 weight ratio, however, was associated with strong inhibition of cytokine production, exceeding that of the peptide alone indicative of a synergistic action (Figure 2, right hand side). Therefore, from these data, use of a combination of antibiotic and peptide in septic patients seems promising.

Furthermore, for therapeutic use, it is important that inhibition of LPS-induced cytokine release also takes place in the presence of proteases that are known to decompose peptides. Therefore, the peptides were incubated in $20 \% \mathrm{AB}$ serum for different time periods. As illustrated in Figure 3, the inhibition of TNF- $\alpha$ production decreased with increasing time of serum incubation; however, even after 2 hours incubation there was still considerable inhibition of cytokine release.

Additional experiments were performed with sequence variants of Pep19-2.5, i.e., the duplicated form, Pep192.5Dup, the variant with all amino acids in a D-configuration (Pep19-2.5D-AA), and a variant in which the final sequence at the C-terminus is lacking (for sequences see Table 1). As demonstrated in Figure 4, duplication of the amino acid sequence did not lead to improvement in the inhibition activity of the peptide, and the peptide with Dconfigured amino acids still had good inhibitory activity. This latter information is important with respect to the activity of proteases in blood serum, which rapidly decompose L-configuration peptides. A further sequence variation, the peptide Pep19-2.5short, lacking the C-terminal hydrophobic sequence FWFWG, led to a near complete abrogation of any inhibitory activity (Figure 5). This finding is of importance since the driving force between the peptides and LPS was shown to be the Coulomb interaction between the basic residues of the peptides ( $\mathrm{R}$ and $\mathrm{K})$, and the negative charges of LPS [33]. In a second step of the interaction, however, intercalation of the C-terminal proximal hydrophobic peptide part into the lipid A hydrophobic moiety was described [34]. As can be deduced from the lack of inhibition of the shortened variant, the latter process has considerable impact on understanding the high binding constant of the Pep19-2.5-LPS interaction [34]. 


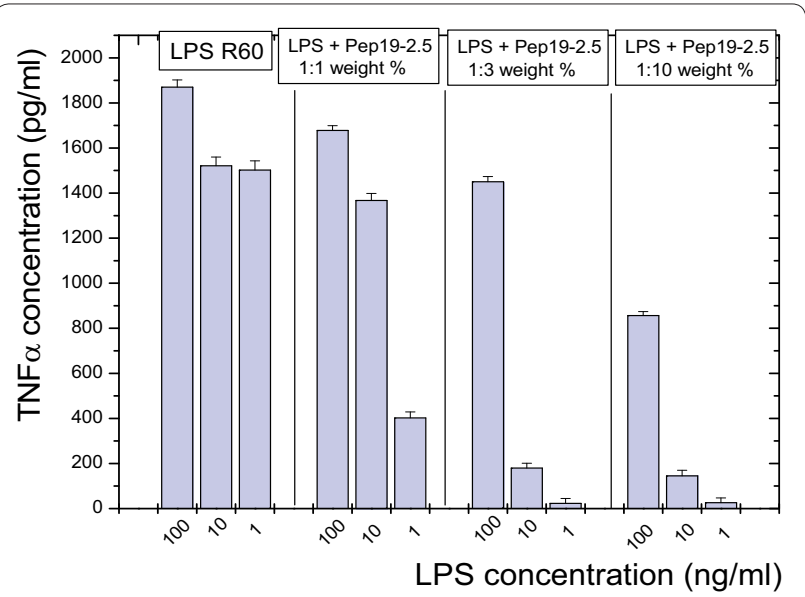

Figure 1. Inhibition in human mononuclear cells of tumornecrosis-factor (TNF)- $a$, induced by lipopolysaccharide (LPS) from Salmonella minnesota Ra (strain R60), by the lead peptide Pep19-2.5 at three different [LPS]:[Pep] weight ratios. Stimulation was performed as described before [34]. Briefly, mononuclear cells (MNC) were isolated from heparinized blood of healthy donors. For

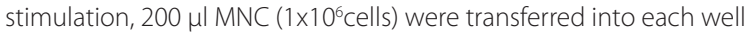
of a 96-well culture plate. LPS R60 and the LPS:peptide mixtures were preincubated for $30 \mathrm{~min}$ at $37^{\circ} \mathrm{C}$, and added to the cultures at $20 \mu \mathrm{l}$ per well and incubated for $4 \mathrm{~h}$ at $37^{\circ} \mathrm{C}$ under $5 \% \mathrm{CO}_{2}$. Supernatants were collected after centrifugation of the culture plates for $10 \mathrm{~min}$ at $400 \mathrm{xg}$ and stored at $-20^{\circ} \mathrm{C}$ until immunological determination of TNF-a, carried out in a Sandwich ELISA using a monoclonal antibody against TNF. The [LPS]:[Pep19-2.5] 1:1 weight\% corresponds to 1.5:1 $\mathrm{M} / \mathrm{M}$, the $1: 3$ weight\% to $1: 2 \mathrm{M} / \mathrm{M}$, and the $1: 10$ weight\% to $1: 6.7$ $M / M$

Therefore, from these and earlier data, the change of lipid A/LPS cubic aggregate into a multilamellar structure is an important step in the ability of the peptides to neutralize endotoxins, associated with an extremely low saturation value of binding at [peptide]:[LPS] $=0.3 \mathrm{M} / \mathrm{M}$, corresponding to the binding saturation of 3 peptides with 10 LPS molecules [33,34]. Thus, human binding proteins, such as LPS binding protein (LBP) and CD14, are impeded from binding to LPS epitopes, the charged head groups, and thus cannot initiate the inflammatory reaction. It has been found, furthermore, that the peptides still exert their effect when they are added in a time-delayed mode, i.e., when they are administered up to 3 hours after LPS addition [36]. This observation is indicative of a membrane process of the interaction, in accordance with recent findings that the peptides readily intercalate into membranes made from phosphatidylcholine and phosphatidylserine as characteristic of eukaryotic cells [34]. After membrane incorporation, the peptides apparently act at the site of membrane receptors such as CD14 and the TLR4/MD2-system, thus competitively inhibiting the interaction with LPS. The details of these processes are currently under investigation. Detailed descriptions of the newest aspects of the use of

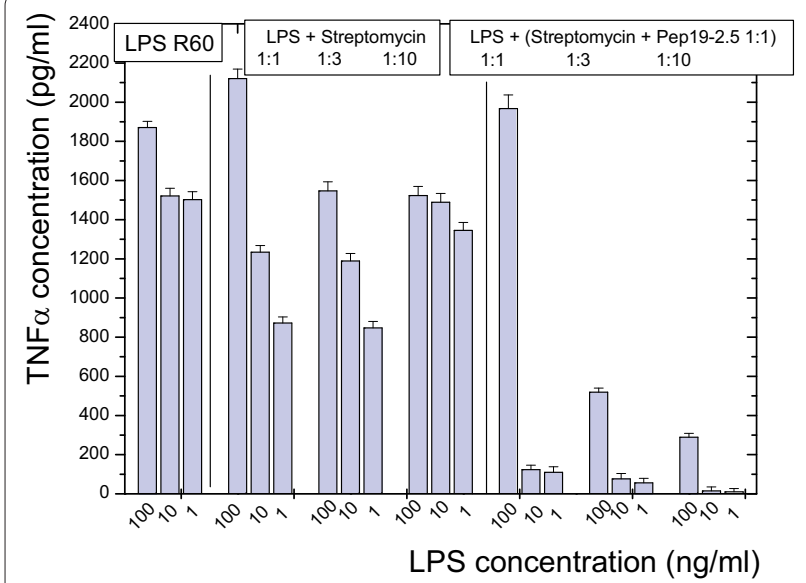

Figure 2. Inhibition in human mononuclear cells of tumor-necrosis-factor (TNF)- $a$, induced by LPS from Salmonella minnesota Ra (strain R60), by streptomycin alone and in combination with the lead peptide Pep19-2.5 (streptomycin:Pep19-2.5 1:1 weight\%) at three different [LPS]:[Streptomycon:Pep] weight ratios. The stimulation was performed as described in the legend of Figure 1.

AMPs compared to other therapeutic approaches are summarized in a recent review [35].

\section{Naturally occurring and synthetic antimicrobial peptides in human and experimental sepsis}

Certain anti-microbial peptides kill bacteria without causing cell disruption. Patients with septic shock, in particular, would benefit because antibiotics are known to promote the liberation of pro-inflammatory cell components and thus augment the severity of septic shock $[37,38]$. A promising therapeutic approach would involve either the combination of AMP analogs with antibiotic therapy or the combination of AMPs with antibiotic drugs. The resultant synergism between the two antimicrobial components may be especially important for the treatment of critically ill patient with severe infections.

Studies on AMPs in patients with severe sepsis or septic shock are limited. LBP and bactericidal/permeability increasing (BPI) protein are comparable with respect to a high-affinity binding domain for the lipid A component of LPS. The difference is that signal transduction is not hampered by LBP, because it binds to CD14 after CD14 has itself formed complexes with endotoxin. In contrast, BPI prevents endotoxin binding to CD14, hence inhibiting cytokine liberation.

In 49 patients with abscesses, peritonitis or uninfected body fluids, LBP and neutrophil granular BPI protein were investigated. In abscesses, compared to peritoneal fluids and non-infected fluids, the BPI/LBP ratio was significantly elevated. Moreover, BPI concentration was increased more in abscesses with Gram-positive compared to those with Gram-negative organisms. BPI may 


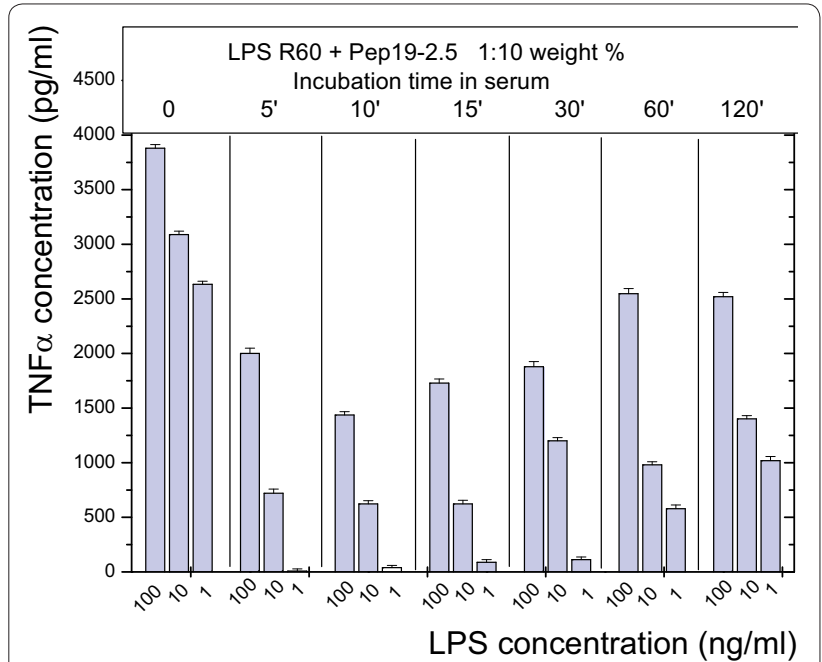

Figure 3. Inhibition in human mononuclear cells of tumornecrosis-factor (TNF)- $a$, induced by LPS from Salmonella minnesota Ra (strain R60), by the lead peptide Pep19-2.5 at three different [LPS]:[Pep] weight ratios. Before addition of the peptide, it was incubated for the given times in $20 \%$ AB serum. The stimulation was performed as described in the legend of Figure 1.

weaken local and systemic effects in response to an inflammatory stimulus induced by endotoxin release [37]. A comparison of BPI bound to the leukocyte surface and BPI and LBP in plasma in healthy volunteers after endotoxin challenge and in patients with Gram-negative sepsis was performed several years ago [39]. In both groups, there was an increase in leukocyte-bound BPI and plasma LBP. Plasma BPI was significantly elevated as a sign of inadequate competition compared to the more frequently detected LBP [39].

A further study investigated BPI levels and BPI/ neutrophil ratios in 42 healthy controls and 34 patients with severe sepsis defined according to the ACCP/SCCM consensus conference [40]. BPI was elevated in sepsis compared to controls (median 15.3, range $<1.6-205 \mu \mathrm{g} / \mathrm{l}$ vs $5.2 ;<1.6-24 \mu \mathrm{g} / \mathrm{l} ; \mathrm{p}<0.001)$. Moreover, sepsis due to Gram-negative compared to Gram-positive pathogens was associated with higher levels of BPI (16.8, range $<1.6-205 \mu \mathrm{g} / \mathrm{l}$ vs. $16.0,<1.6-60 \mu \mathrm{g} / \mathrm{l} ; \mathrm{p}=0.05)$. Because of a possible association with decreased mean arterial pressure, the authors concluded that BPI may reflect the severity of organ dysfunction in sepsis [40].

The development of a recombinant fragment of human BPI (rBPI21) triggered a large study on the therapeutic use of AMPs in pediatric meningococcal infection. Three hundred and ninety-five children with suspected meningococcal sepsis were randomly assigned to receive rBPI21 within 8 hours of diagnosis [41]. Overall, the study failed to demonstrate a significantly reduced mortality after rBPI21 compared to placebo (7.4\% vs. $9.9 \% ; \mathrm{p}=0.48$ ). Adjusted for patients with incomplete infusion of study

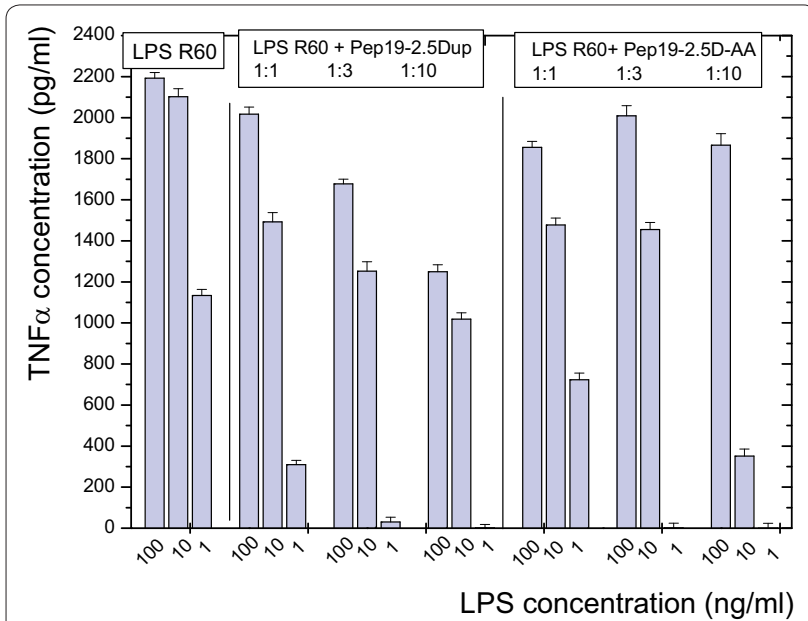

Figure 4. Inhibition in human mononuclear cells of tumornecrosis-factor (TNF)- $\alpha$, induced by LPS from Salmonella minnesota Ra (strain R60), by Pep19-2.5Dup and Pep19-2.5D-AA at three different [LPS]:[Pep] weight ratios. The stimulation was performed as described in the legend of Figure 1.

drug, the mortality was non-significantly lower in the intervention group (rBPI21 2.2\% vs. placebo 6.2\%; $\mathrm{p}=0.07$ ). Furthermore, the rate of amputations decreased non-significantly (rBPI21 3.2\% vs. placebo $7.4 \%$; $\mathrm{p}=0.067)$ and significantly more children regained preillness performance when treated with rBPI21 (77.3\% vs. $66.3 \%$, odds ratio 1.75 [95\% CI 1.08-2.83]; $\mathrm{p}=0.019$ ). The study may have failed to reach its target to reduce mortality because of lower than expected placebo group mortality (9.9\% vs. $25 \%$ expected), but the authors hypothesized that an increased benefit may be possible by using parallel and immediate treatment of rBPI21 and anti-infective agents [41].

A prospective case-control study investigated plasma HBD2 in 16 patients with severe sepsis compared to 18 controls [42]. HBD2 was significantly higher in severe sepsis compared to non-septic critically ill patients and to healthy controls. Additionally, HBD2 gene expression in non-septic patients and healthy controls was significantly higher after induction with LPS compared with sepsis patients. Survival status was independent of the inducibility of HBD2 gene expression. This may be understood as a sign of exhaustion after severe infections and may be related to the complex dysfunction of the innate and adaptive immune system in patients suffering from severe sepsis [43]. Administration of intravenous hydrocortisone as adjunctive therapy in septic shock reduced HBD3 expression in contrast to undisturbed HBD2-inducibility [43].

An additional study in children with sepsis and critically ill control patients revealed that levels of HNP1-3 ( $\alpha$-defensins) were increased with onset of sepsis in plasma of non-neutropenic septic patients (median 


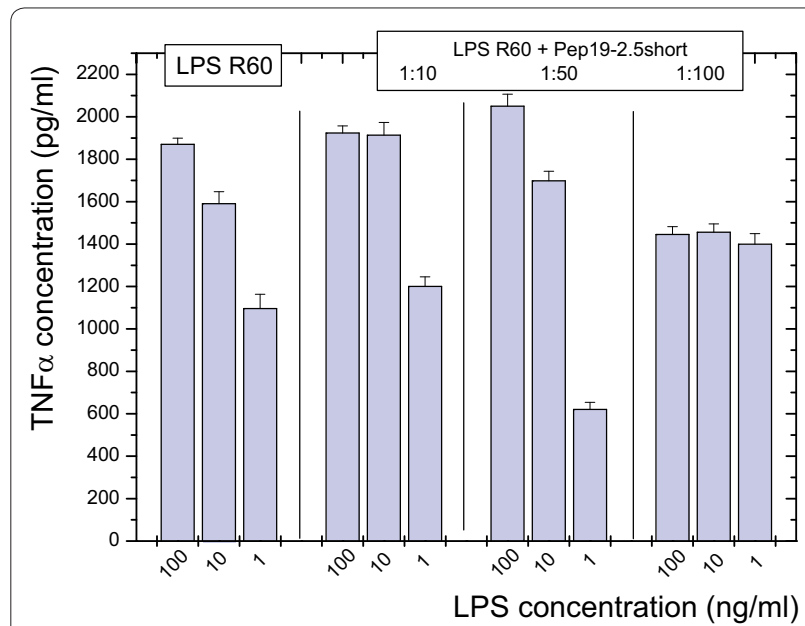

Figure 5. Inhibition of tumor-necrosis-factor-a (TNF) in human mononuclear cells, induced by LPS from Salmonella minnesota Ra (strain R60), by a shortened variant of Pep19-2.5, Pep192.5short (sequence see Table 1), at three different [LPS]:[Pep] weight ratios. The stimulation was performed as described in the legend of Figure 1.

450 [range 194-1031] ng/ml) compared to neutropenic septic patients and controls $(50[0-238] \mathrm{ng} / \mathrm{ml}$ and 150 [0-275] ng/ml; $\mathrm{p}<0.05)$. However, HNP1-3 levels were not related to organ failure or outcome of sepsis [44]. Lactoferrin, derived from neutrophils, was elevated in non-neutropenic sepsis (332 [137-938] ng/ml) whereas lactoferrin in neutropenic sepsis was decreased compared to control patients $(20[0-117] \mathrm{ng} / \mathrm{ml}$ vs 176 [39-312] $\mathrm{ng} / \mathrm{ml} ; \mathrm{p}<0.05)$. Furthermore, lactoferrin was associated with renal and hematologic organ failure. The levels of lactoferrin and HNP1-3 were significantly correlated in this study [44].

Recently, Berkestedt et al. demonstrated that levels of HNP1-3, lactoferrin, BPI and heparin-binding protein (HBP) were higher in 31 patients with sepsis compared to 25 non-septic controls [45]. Out of these AMP, only BPI was associated with outcome, revealing significantly higher levels in non-survivors compared to survivors. The neutrophil granula-derived HBP, BPI and HNP1-3 and lactoferrin were increased in sepsis, thus reflecting the ongoing battle of the innate immunity against invading organisms [45].

The use of polymyxin B and polymyxin E (colistin), cationic polypeptides that neutralize the lethal effects of endotoxin, was abandoned, because nephrotoxicity and neurotoxicity were reported. However, these adverse effects observed in early clinical studies were most likely due to a limited understanding of the pharmacokinetics, pharmacodynamics, and toxicodynamics of these agents, and the use of incorrect quantities [46]. Use was reinstated in a recent study of hemoperfusion with polymyxin-B loaded cartridges in patients with abdominal septic shock (EUPHAS) [47]. In this Italian multicenter trial, 64 patients were assigned to receive standard of care or standard of care plus 2 sessions of polymyxin-B hemoperfusion within 72 hours after the surgical procedure. In addition to improvements in mean arterial pressure and organ dysfunction, 28-day mortality was lower in the polymyxin B group compared to conventional therapy alone (32\% vs. $53 \%$, unadjusted hazard ratio [HR] 0.43 ; 95\% CI 0.20-0.94; adjusted HR, 0.36; 95\% CI 0.16-0.80). In view of the mortality reduction the study was stopped by the ethics committee. To confirm the findings in other patients and to address concerns raised after publication of the EUPHAS study, two further studies are underway (EUPHRATES: clinicaltrials.gov-identifier NCT01046669 and EUPHAS 2 [48]).

Recently, it was demonstrated that a single $25 \mathrm{mg} / \mathrm{kg}$ dose of a synthetic peptide protected mice from infection with $P$. aeruginosa and E. coli [49]. The application of the AMP led to elimination of bacteria in the blood 18 hours after infection, and the bacterial count was significantly lower in other specimens (peritoneal fluid, spleen and liver) compared to control animals. After an additional 22 hours all tested samples were free of bacteria [49]. A further synthetic AMP, named s-thanatin, showed activity against Gram-positive and -negative bacteria [50]. After combination with the antimicrobial peptide it was demonstrated that the minimum inhibitory concentration (MIC) of various antibiotics was decreased by a factor of 2 to 8 (1-3 dilution steps). Moreover, survival after intraperitoneal bacterial challenge was increased after treatment with s-thanatin, in a dose-dependent manner [50]. The most recent study is investigating talactoferrin alfa in a phase III study in patients with severe sepsis (OASIS; clinicaltrials.gov identifier NCT01273779).

\section{Conclusion}

Investigations of the past decade show increasing interest in antimicrobial peptides as a tool for sepsis diagnosis and furthermore as a possible therapeutic intervention. Newly designed peptides with decreased toxicity and a broader range of efficacy may have the potential to provide significant improvements in the treatment of infections.

\section{Abbreviations}

AMP, antimicrobial peptides; BPI, bactericidal/permeability increasing; cfu, colony-forming units; CMV, cytomegalovirus; ENP, endotoxin-neutralizing protein; HBD, human beta-defensins; HBP, heparin-binding protein; HIV,

human immunodeficiency virus; hLF1-11, human lactoferrin 1-11; HNP, human neutrophil peptides; HR, hazard ratio; HSV, herpes simplex virus; IL, interleukin; ITC, isothermal titration calorimetry; LAPF, Limulus anti-LPS factor; LBP, LPS binding protein; LP, lipoprotein; LPS, lipopolysaccharide; MIC, minimum inhibitory concentration; MNC, mononuclear cells; NO, nitric oxide; PMN, polymorphonuclear leukocytes; TNF, tumor necrosis factor.

\section{Competing interests}

KB has applied patent for the structure of a newly developed synthetic antimicrobial peptide: Patent-No:PCT/EP2009/002565. 


\section{Acknowledgements}

The authors are indebted to the German ministry (Ministerium für Bildung und Forschung, BMBF) for financial help in the frame of a preclinical study 'Therapy of infectious diseases with special regards to bacterial sepsis' (project: 01GU0824 and 01GU0826).

\section{Author details}

'Department of Intensive Care, University Hospital, Pauwelsstr. 30, 52074 Aachen, Germany. ${ }^{2}$ Department of Biophysics, Forschungszentrum, Parkallee 10, 23845 Borstel, Germany.

Published: 20 March 2012

\section{References}

1. Rivers E, Nguyen B, Havstad S, et al:: Early goal-directed therapy in the treatment of severe sepsis and septic shock. N Engl J Med 2001 345:1368-1377.

2. Kumar A, Roberts D, Wood KE, et al:: Duration of hypotension before initiation of effective antimicrobial therapy is the critical determinant of survival in human septic shock. Crit Care Med 2006, 34:1589-1596.

3. Vincent $J$, Bernard GR, Beale R, et al:: Drotrecogin alfa (activated) treatment in severe sepsis from the global open-label trial ENHANCE: further evidence for survival and safety and implications for early treatment. Crit Care Med 2005, 33:2266-2277.

4. Valles J, Rello J, Ochagavia A, Garnacho J, Alcala MA: Community-acquired bloodstream infection in critically ill adult patients: impact of shock and inappropriate antibiotic therapy on survival. Chest 2003, 123:1615-1624.

5. Kresken M, Hafner D, Schmitz F-J, Wichelhaus TA, Studiengruppe FD: Resistenzsituation bei klinisch wichtigen Infektionserregern gegenüber Antibiotika in Deutschland und im mitteleuropäischen Raum. Antiinfectives Intelligence. Rheinbach; 2009.

6. Steinstraesser $L$, Kraneburg UM, Hirsch T, et al:: Host defense peptides as effector molecules of the innate immune response: a sledgehammer for drug resistance? Int J Mol Sci 2009, 10:3951-3970.

7. Gordon YJ, Romanowski EG, McDermott AM: A review of antimicrobial peptides and their therapeutic potential as anti-infective drugs. Curr Eye Res 2005, 30:505-515.

8. Skarnes RC, Watson DW: Antimicrobial factors of normal tissues and fluids. Bacteriol Rev 1957, 21:273-294.

9. Shafer WM, Qu X, Waring AJ, Lehrer Rl: Modulation of Neisseria gonorrhoeae susceptibility to vertebrate antibacterial peptides due to a member of the resistance/nodulation/division efflux pump family. Proc Natl Acad Sci USA 1998, 95:1829-1833.

10. Yount NY, Bayer AS, Xiong YQ, Yeaman MR: Advances in antimicrobial peptide immunobiology. Biopolymers 2006, 84:435-458.

11. Peschel A: How do bacteria resist human antimicrobial peptides? Trends Microbiol 2002, 10:179-186.

12. Hancock RE, Sahl HG: Antimicrobial and host-defense peptides as new anti-infective therapeutic strategies. Nat Biotechno/ 2006, 24:1551-1557.

13. Semple F, Webb S, Li HN, et al:: Human beta-defensin 3 has immunosuppressive activity in vitro and in vivo. Eur J Immuno/ 2010, 40:1073-1078

14. Rieg S, Steffen H, Seeber S, et al:: Deficiency of dermcidin-derived antimicrobial peptides in sweat of patients with atopic dermatitis correlates with an impaired innate defense of human skin in vivo. $\mathrm{J}$ Immunol 2005, 174:8003-8010.

15. Meyer JE, Harder J, Gorogh T, et al:: Human beta-defensin-2 in oral cancer with opportunistic Candida infection. Anticancer Res 2004, 24:1025-1030.

16. Milner SM, Ortega MR: Reduced antimicrobial peptide expression in human burn wounds. Burns 1999, 25:411-413.

17. Tao R, Jurevic RJ, Coulton KK, et al:: Salivary antimicrobial peptide expression and dental caries experience in children. Antimicrob Agents Chemother 2005, 49:3883-3888.

18. Lippross S, Klueter T, Steubesand N, et al:: Multiple trauma induces serum production of host defence peptides. Injury, in press.

19. Gryllos I, Tran-Winkler HJ, Cheng MF, et al:: Induction of group A Streptococcus virulence by a human antimicrobial peptide. Proc Natl Acad SciUSA 2008, 105:16755-16760.

20. Berkestedt I, Nelson A, Bodelsson M: Endogenous antimicrobial peptide LL-37 induces human vasodilatation. Br J Anaesth 2008, 100:803-809.

21. Fukumoto K, Nagaoka I, Yamataka A, et al.: Effect of antibacterial cathelicidin peptide CAP18/LL-37 on sepsis in neonatal rats. Pediatr Surg Int 2005, 21:20-24.

22. Torossian A, Gurschi E, Bals R, Vassiliou T, Wulf HF, Bauhofer A: Effects of the antimicrobial peptide LL-37 and hyperthermic preconditioning in septic rats. Anesthesiology 2007, 107:437-441.

23. Zhang L, Falla TJ: Antimicrobial peptides: therapeutic potential. Expert Opin Pharmacother 2006, 7:653-663.

24. Giles FJ, Rodriguez R, Weisdorf D, et al:: A phase III, randomized, doubleblind, placebo-controlled, study of iseganan for the reduction of stomatitis in patients receiving stomatotoxic chemotherapy. Leuk Res 2004, 28:559-565.

25. Vallespi MG, Glaria LA, Reyes O, Garay HE, Ferrero J, Arana MJ: A Limulus antilipopolysaccharide factor-derived peptide exhibits a new immunological activity with potential applicability in infectious diseases. Clin Diagn Lab Immunol 2000, 7:669-675

26. Ried C, Wahl C, Miethke T, et al:: High affinity endotoxin-binding and neutralizing peptides based on the crystal structure of recombinant Limulus anti-lipopolysaccharide factor. J Bio/ Chem 1996, 271:28120-28127.

27. Andrä J, Lamata M, Martinez de Tejada G, Bartels R, Koch MH, Brandenburg K Cyclic antimicrobial peptides based on Limulus anti-lipopolysaccharide factor for neutralization of lipopolysaccharide. Biochem Pharmacol 2004, 68:1297-1307

28. Andrä J, Garidel P, Majerle A, et al.: Biophysical characterization of the interaction of Limulus polyphemus endotoxin neutralizing protein with lipopolysaccharide. Eur J Biochem 2004, 271:2037-2046.

29. Andrä J, Howe J, Garidel P, et al: Mechanism of interaction of optimized Limulus-derived cyclic peptides with endotoxins: thermodynamic, biophysical and microbiological analysis. Biochem J 2007, 406:297-307.

30. Brandenburg K, Schromm AB, Gutsmann T: Endotoxins: Structure, function, and recognition: Relationship between structure, function, and activity. Subcell Biochem 2010, 53:53-67.

31. Pan CY, Chao TT, Chen JC, et al:: Shrimp (Penaeus monodon) antilipopolysaccharide factor reduces the lethality of Pseudomonas aeruginosa sepsis in mice. Int Immunopharmacol 2007, 7:687-700.

32. Kowalski I, Kaconis Y, Andrä J, et al.: Physicochemical and biological characterization of anti-endotoxin peptides and their influence on lipid properties. Protein Pept Lett 2010, 17:1328-1333.

33. Gutsmann T, Razquin-Olazaran I, Kowalski I, et al.: New antiseptic peptides to protect against endotoxin-mediated shock. Antimicrob Agents Chemother 2010, 54:3817-3824.

34. Kaconis Y, Kowalski I, Howe J, et al.: Biophysical mechanisms of endotoxin neutralization by cationic amphiphilic peptides. Biophys J 2011, 100:2652-2661.

35. Brandenburg K, Andrä J, Garidel P, Gutsmann T: Peptide-based treatment of sepsis. Appl Microbiol Biotechnol 2011, 90:799-808.

36. Martinez de Tejada G, Sánchez-Gómez S, Razquin-Olazaran I, et al:: Bacterial cell wall compounds as promising targets of antimicrobial agents I. Antimicrobial peptides and lipopolyamines. Curr Drug Targets, in press.

37. Opal SM, Palardy JE, Marra MN, Fisher CJ Jr, McKelligon BM, Scott RW: Relative concentrations of endotoxin-binding proteins in body fluids during infection. Lancet 1994, 344:429-431.

38. Marra MN, Wilde CG, Griffith JE, Snable JL, Scott RW: Bactericidal/ permeability-increasing protein has endotoxin-neutralizing activity. J Immunol 1990, 144:662-666

39. Calvano SE, Thompson WA, Marra MN, et al.: Changes in polymorphonuclear leukocyte surface and plasma bactericidal/permeability-increasing protein and plasma lipopolysaccharide binding protein during endotoxemia or sepsis. Arch Surg 1994, 129:220-226.

40. Rintala E, Peuravuori H, Pulkki K, Voipio-Pulkki LM, Nevalainen T: Bactericidal/ permeability-increasing protein (BPI) in sepsis correlates with the severity of sepsis and the outcome. Intensive Care Med 2000, 26:1248-1251.

41. Levin M, Quint PA, Goldstein B, et al: Recombinant bactericidal/ permeability-increasing protein (rBPI21) as adjunctive treatment for children with severe meningococcal sepsis: a randomised trial. rBPI21 Meningococcal Sepsis Study Group. Lancet 2000, 356:961-967.

42. Duits LA, Rademaker M, Ravensbergen B, et al:: Inhibition of hBD-3, but not hBD-1 and hBD-2, mRNA expression by corticosteroids. Biochem Biophys Res Commun 2001, 280:522-525.

43. Book M, Chen $\mathrm{Q}$, Lehmann LE, et al:: Inducibility of the endogenous antibiotic peptide beta-defensin 2 is impaired in patients with severe sepsis. Crit Care 2007, 11:R19. 
44. Thomas NJ, Carcillo JA, Doughty LA, Sasser H, Heine RP: Plasma concentrations of defensins and lactoferrin in children with severe sepsis. Pediatr Infect Dis J 2002, 21:34-38.

45. Berkestedt I, Herwald H, Ljunggren L, Nelson A, Bodelsson M: Elevated plasma levels of antimicrobial polypeptides in patients with severe sepsis. J Innate Immun 2010, 2:478-482.

46. Li J, Nation RL, Turnidge JD, et al.: Colistin: the re-emerging antibiotic for multidrug-resistant Gram-negative bacterial infections. Lancet Infect Dis 2006, 6:589-601.

47. Cruz DN, Antonelli M, Fumagalli R, et al.: Early use of polymyxin B hemoperfusion in abdominal septic shock: the EUPHAS randomized controlled trial. JAMA 2009, 301:2445-2452.

48. Martin EL, Cruz DN, Monti G, et al.: Endotoxin removal: how far from the evidence? The EUPHAS 2 Project. Contrib Nephrol 2010, 167:1 19-125.
49. Pini A, Falciani C, Mantengoli E, et al:: A novel tetrabranched antimicrobial peptide that neutralizes bacterial lipopolysaccharide and prevents septic shock in vivo. FASEB J 2010, 24:1015-1022.

50. Wu G, Fan X, Li L, et al.: Interaction of antimicrobial peptide s-thanatin with lipopolysaccharide in vitro and in an experimental mouse model of septic shock caused by a multidrug-resistant clinical isolate of Escherichia coli. Int J Antimicrob Agents 2010, 35:250-254.

doi: $10.1186 / \mathrm{cc} 11220$

Cite this article as: SchuerholzT, et al:: Antimicrobial peptides and thei potential application in inflammation and sepsis. Critical Care 2012, 16:207. 